\title{
Promoter methylation is not associated with $F L C N$ irregulation in lung cyst lesions of primary spontaneous pneumothorax
}

\author{
YIBING DING ${ }^{1,2^{*}}$, WEI ZOU $^{3 *}$, CHENGCHU ZHU $^{4}$, HAIYAN MIN $^{1,2}$, DEHUA MA $^{4}$, BAOFU CHEN $^{4}$, \\ MINHUA YE ${ }^{4}$, YANQING PAN $^{3}$, LEI CAO $^{3}$, YUEMING WAN $^{3}$, QIUXIANG ZHU ${ }^{1}$, HAIZHEN XIA $^{1}$, \\ WENWEN ZHANG ${ }^{1}$, YING FENG ${ }^{1}$, QIAN GAO $^{1,2}$ and LONG YI $^{1,2}$ \\ ${ }^{1}$ Center for Translational Medicine; ${ }^{2}$ Jiangsu Key Laboratory for Molecular Medicine, Nanjing University Medical School; \\ ${ }^{3}$ Department of Thoracic Surgery, Nanjing Chest Hospital, Nanjing, Jiangsu 210093; ${ }^{4}$ Department of Cardiothoracic \\ Surgery, Taizhou Hospital of Zhejiang, Wenzhou Medical University, Linhai, Zhejiang 317000, P.R. China
}

Received November 25, 2014; Accepted August 25, 2015

DOI: $10.3892 / \mathrm{mmr} .2015 .4341$

\begin{abstract}
Germline mutations in FLCN are responsible for $\sim 10 \%$ of patients with primary spontaneous pneumothorax (PSP), characterized by multiple lung cysts in the middle/lower lobes and recurrent pneumothorax. These clinical features are also observed in a substantial portion of patients with sporadic PSP exhibiting no FLCN coding mutations. To assess the potential underlying mechanisms, 71 patients with PSP were selected, including 69 sporadic and 2 familial cases, who bared $F L C N$ mutation-like lung cysts, however, harbored no FLCN protein-altering mutations. Notably, in a significant proportion of the patients, FLCN irregulation was observed at the transcript and protein levels. Genetic analyses of the cis-regulatory region of FLCN were performed by sequencing and multiplex ligation-dependent probe amplification assay. No inheritable DNA defect was detected, with the exception of a heterozygous deletion spanning the $F L C N$ promoter, which was identified in a family with PSP. This mutation caused a reduction in the expression of FLCN in the lung cysts. Pedigree analysis demonstrated that haploinsufficiency of FLCN was pathogenic. To determine whether epigenetic mechanisms may be involved in the irregulation of $F L C N$, the promoter methylation status was measured in the remainder of the patients. No evidence of $F L C N$ promoter methylation was demonstrated. The present study suggested that $F L C N$ irregulation in lung cysts of PSP is not associated with promoter methylation.
\end{abstract}

Correspondence to: Dr Long Yi or Professor Qian Gao, Center for Translational Medicine, Nanjing University Medical School, 22 Hankou Road, Nanjing, Jiangsu 210093, P.R. China

E-mail: yilong@nju.edu.cn

E-mail: qian_gao@nju.edu.cn

${ }^{*}$ Contributed equally

Key words: FLCN gene, primary spontaneous pneumothorax, gene expression, promoter methylation

\section{Introduction}

Primary spontaneous pneumothorax [PSP; Online Mendelian Inheritance in Man (OMIM)\#173600] occurs in patients without clinically apparent underlying lung disease. Of PSP cases, $\sim 10 \%$ are caused by germline mutations in the FLCN gene $(1,2)$, which is known to cause Birt-Hogg-Dube syndrome (BHD; OMIM\#135150), an autosomal dominant condition characterized by skin fibrofolliculomas, pulmonary cysts/spontaneous pneumothorax and renal cancers $(3,4)$. FLCN mutation may result in isolated PSP with no skin or renal manifestations, presumably due to an incomplete penetration, as has been reported in multiple previous case studies $(1,2,5-7)$.

Lung cysts are the common pathogenic ground of PSP $(8,9)$. Multiple lung cysts in patients with FLCN mutations are often observed randomly and bilaterally distributed in the lung, particularly in its lower portion (10-13). By contrast, apical bullae are often observed in patients with sporadic PSP without FLCN mutations (14-16). Notably, $F L C N$ mutation-like lung cysts are also observed in a significant portion of non-FLCN mutant sporadic PSP cases (6). Whether these cases are in any way associated with $F L C N$ disruption remains to be elucidated.

The FLCN gene (OMIM\#607273) encodes an evolutionarily conserved protein, folliculin, with no apparent functional motif currently recognized. The majority of the pathogenic FLCN mutations, identified to date, resulted in premature truncation of the protein (http://www.skingenedatabase.com/) (17). FLCN missense mutations and small in-frame deletions were reported to predominantly disrupt protein stability and lead to significant reductions in the expression of FLCN (18). No evidence for a dominant negative effect of $F L C N$ mutants was observed in the transfected cells. Additionally, large intragenic deletions spanning the putative $F L C N$ promoter region have been reported in families with BHD. Luciferase reporter assays demonstrated that a deletion of the putative promoter dramatically reduced the gene expression in vitro (19). In addition, FLCN inactivation caused by promoter methylation has also been detected in types of renal tumor $(20,21)$. This raised the possibility 
that epigenetic regulation of $F L C N$ may contribute to the pathology of BHD.

The present study selected 71 patients with PSP, who harbored a $F L C N$ mutation-like lung phenotype, however, exhibited no germline and somatic mutations in the FLCN coding regions. Significant variations in the expression of $F L C N$ were observed in the lung cysts of these patients, when compared with those of the patients with BHD and the controls. It was hypothesized that transcriptional irregulation of FLCN may be an important mechanism contributing to the development of lung cysts and, subsequently, PSP. The present study aimed to search for epigenetic variations in the putative promoter of FLCN in the patients with PSP.

\section{Materials and methods}

Patients. The present study was approved by the ethics committees of Nanjing University Medical School, Nanjing Chest Hospital and Taizhou Hospital of Zhejiang Province. The 71 selected patients with PSP included 69 sporadic patients and 2 patients with a family history. The patients were clinically diagnosed with PSP on the basis of a thorax computed tomography scan and underwent surgeries for the treatment of pneumothorax at two tertiary hospitals, Taizhou Hospital of Zhejiang Province and Nanjing Chest Hospital. The patients were enrolled for the present study since they exhibited FLCN-like multiple lung cysts, however, exhibited no mutations in the $F L C N$ coding region. A thorough screen for skin and renal abnormalities was performed by cutaneous examination and abdominal ultrasonography, respectively. Peripheral blood samples and tissue samples derived from the clinically resected lung lesions were collected. The control group included 11 morphologically normal lung tissues, which were obtained from patients with stage I non-small cell lung carcinomas that underwent lobectomy. These samples were obtained at least $5 \mathrm{~cm}$ from the tumor locus. Written informed consent was obtained from all patients involved in the present study.

DNA sequencing. Genomic DNA was extracted using the DNeasy Blood and Tissue kit (Qiagen, Hilden, Germany). The FLCN exons and flanking intron regions were sequenced, as previously reported (1). The PCR products were amplified using the BigDye Terminator kit (Applied Biosystems Life Technologies, Foster City, CA, USA) and sequenced on an ABI 3130 Genetic Analyzer (Applied Biosystems Life Technologies). The data were analyzed by referring to the reference sequence (NM_144997.5) obtained from the NCBI database (http://www.ncbi.nlm.nih.gov/gene/201163\# reference-sequences).

Haplotype analysis. Haplotype analysis was performed in the family of patient F260. A total of 16 individuals were genotyped, among which 7 were affected and 9 were unaffected. Eight microsatellite markers were used, which spanned an 11.4-cM distance flanking the FLCN locus on chromosome 17, including D17S799, D17S921, D17S122, D17S1857, D17S740, D17S2196, D17S2187 and D17S798. Haplotypes were determined from the genotype order in which the least number of recombinants occurred.
Multiplex ligation-dependent probe amplification assay (MLPA). A total of two sets of MLPA assays were used to detect deletions/duplications in FLCN and its up/downstream regions. The commercial kit, P256-B1 FLCN (MRC-Holland, Amsterdam, Netherlands), contained probes targeting all 14 exons. Another MLPA kit was synthesized (available on request) with 15 probes targeting $9 \mathrm{~kb}$ upstream and $5 \mathrm{~kb}$ downstream of the gene. The MLPA reactions were performed, according to the manufacturer's instructions. The data were analyzed using the Coffalyser, NET software (version 9.0; MRC-Holland). The patient data was normalized against those of the control individuals. Threshold values were set at 0.7-1.4 for normal, $<0.4$ for deletion and $>1.5$ for duplication.

Reverse transcription-quantitative polymerase chain reaction $(R T-q P C R)$. The total RNA was extracted from the lung tissues using an RNeasy Mini kit (Qiagen). The RNA was reverse transcribed into cDNA using PrimeScript RT reagent kit (Takara Bio, Inc., Dalian, China). The mRNA expression of FLCN was determined using FastStart TaqMan Probe Master (Roche, Basel, Switzerland) on a StepOne Real-Time PCR system (Applied Biosystems Life Technologies). Probe \#63 (cat. no. 4688627001; Roche) and the intron-spanning primer pair, Forward: 5'-GGACCAGTGCCTCGTCTG-3' and reverse: 5'-GGTGAACTTAAAAAGCACCTTTCA-3', were selected for FLCN. GAPDH was selected as a control gene for normalization. The data were analyzed using the $2^{-\Delta \Delta C t}$ method (22). Each sample was run in triplicate and all reactions were performed twice.

Western blot analysis. Lung cyst samples were homogenized using a dounce homogenizer (Corning, Shanghai, China) and suspended in ice cold lysis buffer containing protease inhibitors (Beyotime Institute of Biotechnology, Shanghai, China). The homogenates were centrifuged at 13,000 x $\mathrm{g}$ for $20 \mathrm{~min}$ at $4^{\circ} \mathrm{C}$. The protein concentrations of the supernatants were quantified using a BCA Protein Assay kit (Beyotime Institute of Biotechnology). A total of $100 \mathrm{mg}$ protein was separated by $12 \%$ SDS-PAGE and transferred onto immobilon membranes (Millipore, Bedford, MA, USA). The membranes were blocked with $5 \%$ non-fat milk in Tris-buffered saline with $0.05 \%$ Tween 20 (TBST; Millipore) at room temperature and then washed with TBST for 15 min three times. The membranes were incubated overnight at $4^{\circ} \mathrm{C}$ with the primary antibodies rabbit anti-FLCN (D14G9) monoclonal antibody (mAb) (cat no. 3697; Cell Signaling Technology, Beverly, MA, USA; $1: 1,000$ dilution) and rabbit $\beta$-actin (13E5) mAb (cat no. 4970; Cell Signaling Technology; 1:1,000 dilution). Blots were washed in TBST for $15 \mathrm{~min}$ three times and subsequently incubated with horseradish peroxidase-conjugated goat antirabbit immunoglobulin G (cat no. ab6721; Abcam, Cambridge, UK; 1:3,000 dilution). Immunoreactive bands were detected using an eECL western blot kit (CWBio, Beijing, China). The images of the bands were captured using a digital G-box chemiluminescent imaging system (Syngene, Frederick, MD, USA). Each sample was analyzed at least twice.

Methylation analysis. The $\mathrm{CpG}$ island region in the FLCN gene was predicted using the University of California Santa 


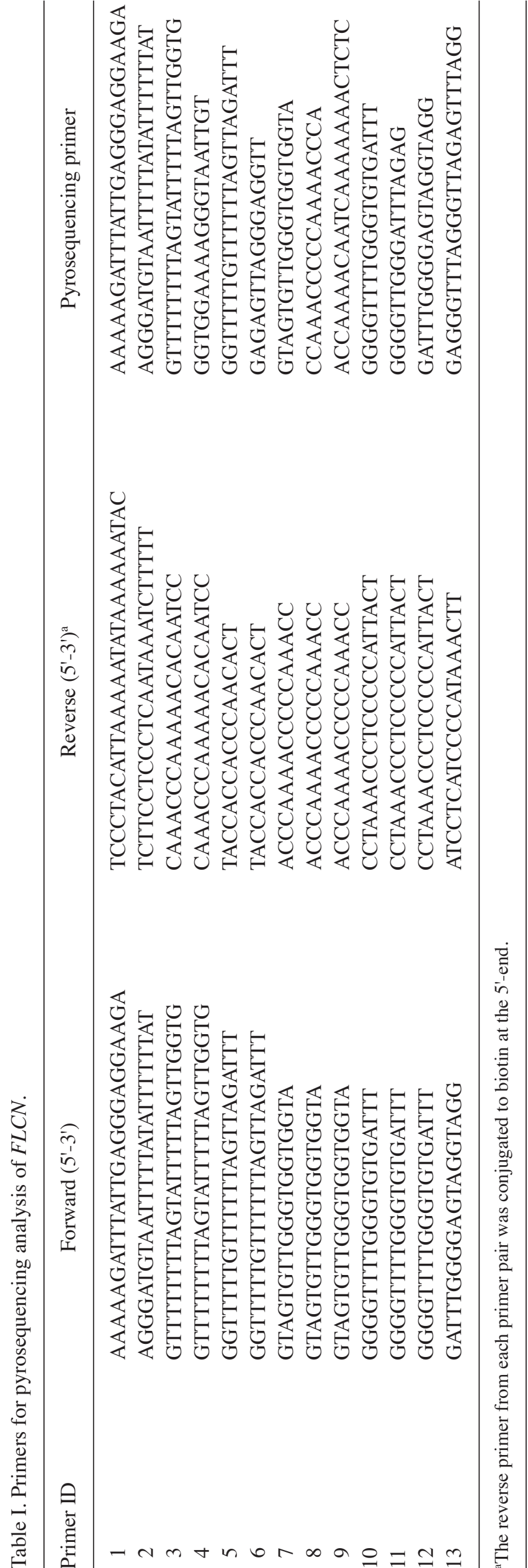

Table II. mRNA and protein expression levels of $L C N$ in 71 patients with PSP without $F L C N$ coding mutations.

\begin{tabular}{lccc}
\hline Group & $\mathrm{n}$ & mRNA level & Protein level \\
\hline$F L C N$-low PSP & 25 & $0.37 \pm 0.11$ & $0.39 \pm 0.40$ \\
$F L C N$-normal PSP & 37 & $1.05 \pm 0.29$ & $0.92 \pm 0.57$ \\
$F L C N$-high PSP & 9 & $2.83 \pm 0.54$ & $2.02 \pm 1.12$ \\
Control lung tissue & 11 & $1.06 \pm 0.47$ & $1.01 \pm 0.25$ \\
\hline
\end{tabular}

PSP, primary spontaneous pneumothorax.

Cruz (UCSC) genome browser (http://genome.ucsc.edu) and CpGplot software (version EMBOSS 6.6.0.0; http://www.ebi. ac.uk). A panel of 13 pyrosequencing assays were performed to quantify the methylation value of $69 \mathrm{CpG}$ islands for each sample. The primers for amplification and pyrosequencing were designed using PyroMark assay design software v.2.0 (Qiagen) (Table I). A total of $500 \mathrm{ng}$ DNA sample was treated with EZ DNA methylation-Gold kit (Zymo Research, Orange, CA, USA), and the converted DNA was amplified using a PyroMark PCR kit (Qiagen), according to the manufacturer's instruction. The PCR products were pyrosequenced and the data were analyzed on a PyroMark Q96 ID (Qiagen). A value of $6 \%$ was set as a convincing absolute threshold for methylation, according to manufacturer's instructions and suggestions from a previous study (23).

Combined bisulphite restriction analysis (COBRA) was further performed, as described previously (24). A $281 \mathrm{bp}$ sequence within the $\mathrm{CpG}$ islands, including $26 \mathrm{CpGs}$ (CpG28-53), were amplified by semi-nested PCRs with no bias towards methylated or unmethylated templates. The PCR products were treated with the restriction endonucleases, TaqI (Promega, Madison, WI, USA) and BstUI (BioLabs, Ipswich, MA, USA), and were subsequently separated by gel electrophoresis to detect the digestion products. The universal methylated human DNA standard (Zymo Research) was used as a positive control.

Statistical analysis. All statistical analyses were performed using the SPSS statistics 17.0 software (SPSS, Inc., Chicago, IL, USA). For mRNA expression, the statistical significance was determined using unpaired, two-tailed Student's t-test. For the DNA methylation status, the statistical significance was evaluated using the Mann-Whitney U-test. $\mathrm{P}<0.05$ was considered to indicate a statistically significant difference.

\section{Results}

FLCN irregulation is observed in lung cysts of PSP. To determine whether the $F L C N$ mutation-like lung phenotype was associated with an alteration in the expression of $F L C N$, the mRNA expression levels of $F L C N$ in lung cyst lesions from the 71 patients with PSP were determined. A wide-spread distribution of the mRNA expression levels of FLCN was observed, when compared with those of the BHD and control groups (Table II; Fig. 1A). As seen in Fig. 1A, the mRNA expression levels of $F L C N$ in lung cyst lesions from the 


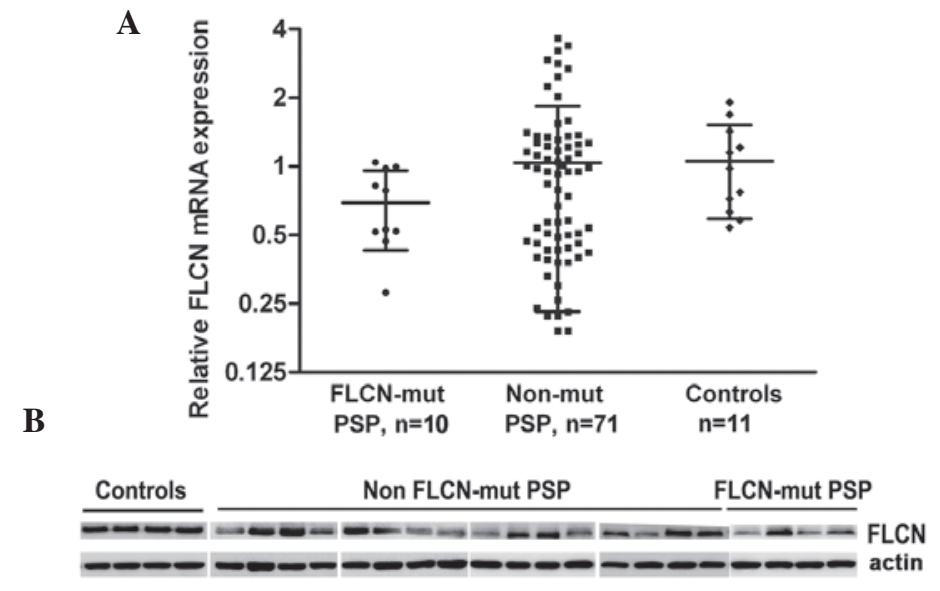

Figure 1. Altered expression levels of $F L C N$ in 71 patients with PSP, without $F L C N$ mutations. (A) The relative mRNA expression levels of $F L C N$ in the patient group (71 patients with PSP, without mutations in $F L C N$ coding region), FLCN-mutant group (10 patients with PSP with $F L C N$ germline mutations) and in the control group (11 morphologically normal lung tissues). Horizontal lines indicate the mean \pm standard deviation. (B) Representative western blot analyses of the protein expression levels of $F L C N$ in the patients and controls. PSP, primary spontaneous pneumothorax.

patients with BHD were reduced $(0.69 \pm 0.26)$, suggesting that certain mutant transcripts may be unstable. Notably, the levels of the FLCN transcript from the 71 patients with PSP may be divided in to three groups: Low, medium and high. About one third of the patients with PSP $(35.2 \%, 25 / 71)$ exhibited a 2-5 fold decrease in the mRNA expression of FLCN, with the group average of $0.37 \pm 0.11$, when compared with that of the control group (1.06 \pm 0.47$)$. Additionally, $\sim 12.7 \%$ patients (9/71) revealed an increase in the mRNA expression of FLCN in the cyst lesions (2.83 \pm 0.54$)$. Similar changes were observed on the protein expression of FLCN (Table II; Fig. 1B). In 80\% of the patients, the tendency of the changes in the mRNA and protein expression levels of FLCN was consistent. This data argued that FLCN irregulation may be associated with the pathogenesis of lung cysts in the patients with sporadic PSP.

Hypoid insufficiency of FLCN is pathogenic. To investigate the mechanism of $F L C N$ irregulation in the patients with PSP, the sequence variations in the cis-regulatory region of $F L C N$ were initially identified. A $1.6 \mathrm{~kb}$ upstream fragment, including the 5 '-flank (650 bp), non-coding exon 1 (228 bp) and partial intron 1 (700 bp) of $F L C N$, was sequenced. No sequence mutation was identified in any of the 71 patients. A total of three common single nucleotide polymorphisms were confirmed, including rs1708629, rs1736209 and rs1736208.

The samples were subsequently investigated by MLPA analysis for large deletions/duplications. An FLCN intragenic deletion was detected in patient F260, with a positive family history. Pedigree and haplotype analysis revealed that the deletion co-segregated among the affected individuals in the family (Fig. 2A). PCR amplification and bidirectional sequencing of the junction fragment revealed a 7,543 bp deletion, including the non-coding exons $1-3$ and a $1.3 \mathrm{~kb}$ upstream sequence (Fig. 2B). The deletion resulted in the removal of the putative $F L C N$ promoter region. All family members harboring the deletion exhibited multiple lung cysts, basally located or randomly distributed on the lung (Fig. 2C-E), and two experienced pneumothorax. These phenotypic features were similar with the clinical manifestations of patients with pathogenic FLCN mutations.
To confirm that this heterozygous deletion disrupted the transcription of the mutant allele of FLCN, the expression of $F L C N$ in patient $\mathrm{F} 260$ was determined. About a 50\% reduction in the level of $F L C N$ was observed in lung cyst lesions from patient F260 at the mRNA (47.4\%) and protein (43.5\%) expression levels, as compared with those of the controls (Fig. 2F and G). These results suggested that FLCN insufficiency caused by the heterozygous FLCN promoter deletion is pathogenic in the development of lung cysts.

Smallest region of overlap (SRO) for the FLCN promoter deletions contains $C p G$ islands. The FLCN promoter appeared to be a recombination hotspot, in which several large deletions were identified in families with BHD (19). Within the SRO (1,893 bp) for all deletions, a CpG-enriched sequence was predicted by the UCSC genome browser and CpGPlot software (Fig. 3A). The $\mathrm{CpG}$ islands, located -628 to +335 of the transcription starting site, encompass the 5'-flank, exon 1 and partial intron 1, with $72 \mathrm{CpG}$ loci (Fig. 3B). This region is predicted to contain multiple putative regulatory elements, including Sp1, AP-2, GCF and Early-Seq1 (Promoter Scan software, http://www-bimas.cit. nih.gov). It was speculated that the methylation status of the $\mathrm{CpG}$ islands may potentially affect the binding of transcription factors and consequently result in the alteration of the expression of FLCN.

Promoter methylation is not associated with FLCN irregulation. The methylation status of the $F L C N$ promoter in 70 patients and 11 controls was quantitatively analyzed by pyrosequencing. No statistical difference in the methylation pattern of the FLCN CpG islands was observed between different groups of patients with PSP and the controls. Dense methylation across the $\mathrm{CpG}$ islands was not observed in any sample analyzed, neither patients nor the controls (Fig. 3C). For each sample, $>95 \%$ of the $\mathrm{CpG}$ loci were unmethylated. Low methylation values, often $<15 \%$, at seldom individual loci $(\mathrm{CpG} 38,49,53)$ were detected in a few samples, however, none were demonstrated to be associated with the expression of FLCN. The methylated CpGs were often separated 
A

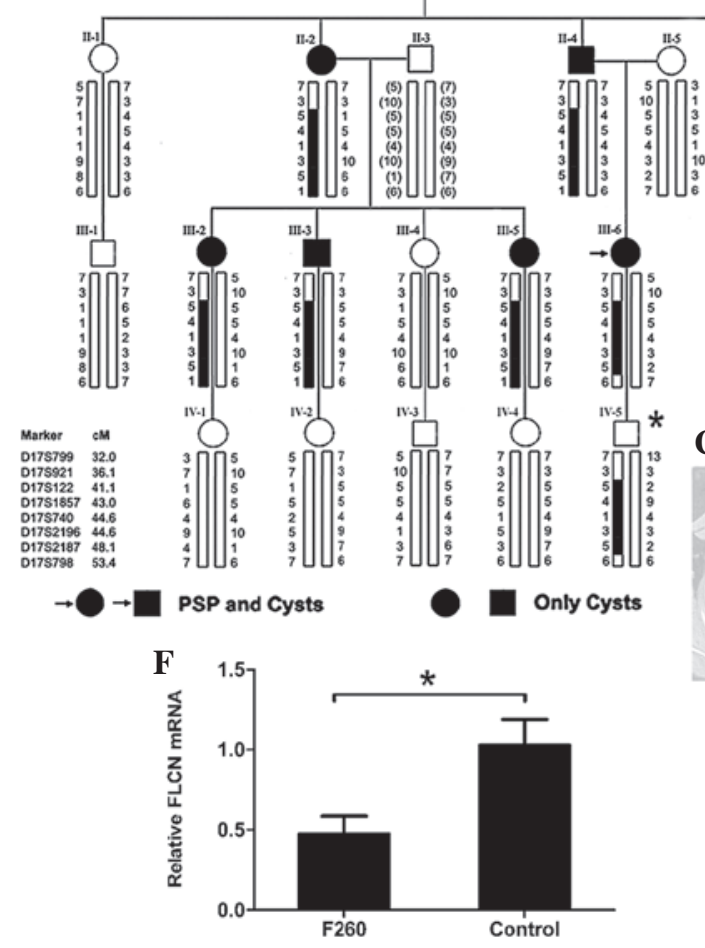

B

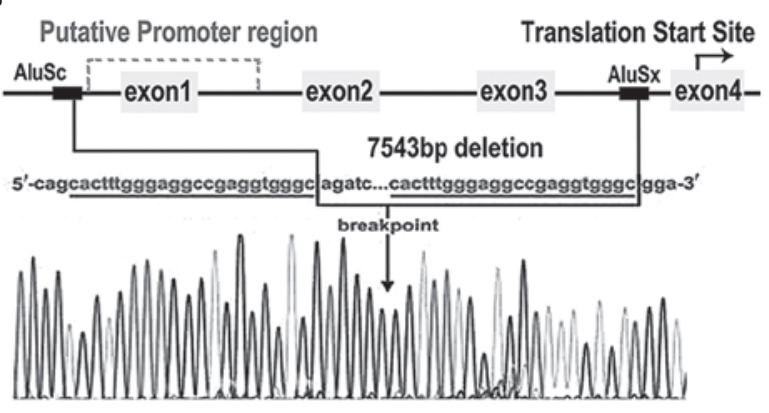

D

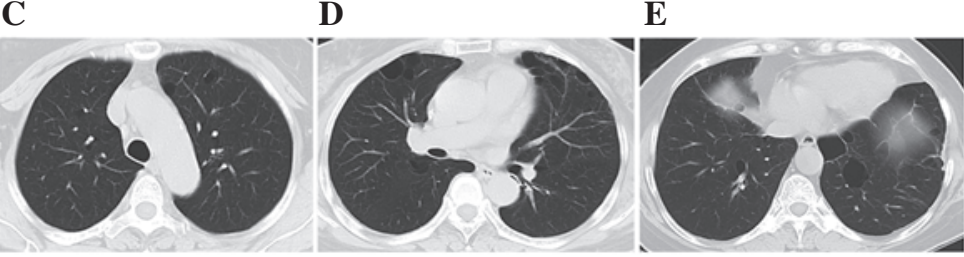

G

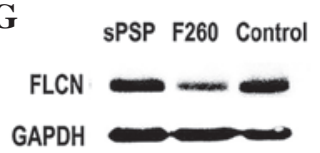

Figure 2. A heterozygous $F L C N$ promoter deletion identified in a family with PSP. (A) Pedigree and haplotype analyses of the PSP family. *A 2-year-old deletion carrier (IV-5) received no clinical examinations. (B) The 7,543 bp deletion mediated by the microhomology in Alu repeats spanned the $F L C N$ promoter region. Computed tomography images of a deletion carrier (II-4) demonstrated multiple bilateral lung cysts of various sizes and shapes, located in the (C) upper, (D) medial and (E) lower zones. (F) Patient F260 (III-6) exhibited a 50\% reduction in the mRNA expression of FLCN as compared with that of the control ( ${ }^{*} \mathrm{P}=0.0001$; the data are presented as the mean \pm standard deviation). (G) A consistent reduction in protein expression was detected in F260. sPSP, sporadic PSP patient; control, morphologically normal lung tissues; PSP, primary spontaneous pneumothorax.

by neighboring unmethylated CpGs. In short, no correlation between methylation levels and the expression of FLCN was established.

To further confirm the pyrosequencing results, COBRA methylation analysis of the $\mathrm{CpG}$ islands was performed in all patients and controls. The $281 \mathrm{bp}$ amplified region (CpG28-53) included two TaqI sites (tcga) and Bst UI (cgcg) sites, however, following bisulphite treatment, no digestion products were observed in any sample. The absence of the digestion products of restriction endonucleases demonstrated that the originally unmethylated sites were lost due to bisulphite conversion. All the $\mathrm{CpG}$ loci analyzed were unmethylated.

\section{Discussion}

PSP is a clinical hallmark of BHD syndrome, since lung cysts/spontaneous pneumothorax are the most frequent and the initial presenting manifestation compared with the skin or renal features (10-12). Up to $80 \%$ of patients with BHD were identified to exhibit multiple lung cysts, and $24 \%$ experienced pneumothorax. Our previous study demonstrated that $\sim 10 \%$ of PSP cases are caused by germline mutations in the FLCN gene (2). However, more sporadic PSP cases with FLCN-like lung cysts, however, no FLCN mutations, were observed. Whether an FLCN-associated mechanism was involved in these cases, and in addition, whether a mechanism other than genetic defect, including epigenetic alteration, is responsible for the development of FLCN-like lung cysts are interesting and worthy of further investigations.

Previous studies of BHD are predominantly focused on the germline mutations in the FLCN coding region. In the present study, a heterozygous FLCN promoter deletion was identified in a family with PSP exhibiting characteristic $F L C N$-like lung cysts. A reduced expression of FLCN was observed in the lesion tissues, consistent with a previous study, which assessed the FLCN promoter function in vitro (19). The present study provided further evidence to support the notion that the downregulation of FLCN is pathogenic in lung cyst formation. Epigenetic mechanisms have long been associated with gene irregulation and human disease (25). In renal tumors, the involvement of FLCN promoter methylation has been hypothesized $(20,21)$, however, inconsistent observations were reported $(24,26)$. No previous studies have investigated the association between FLCN promoter methylation and the development of lung cysts. The present study observed significant variability in the expression of FLCN in the lung lesions of patients with non-FLCN mutant sporadic PSP, however, demonstrated no evidence for the association of $F L C N$ promoter methylation with these cases. The lack of FLCN promoter methylation in patients with PSP with FLCN-like lung cysts is a valuable observation, which provided evidence to refocus our future research. Future studies targeting both genetic and epigenetic mechanisms are required to elucidate the molecular nature of $F L C N$ irregulation in PSP. 
A

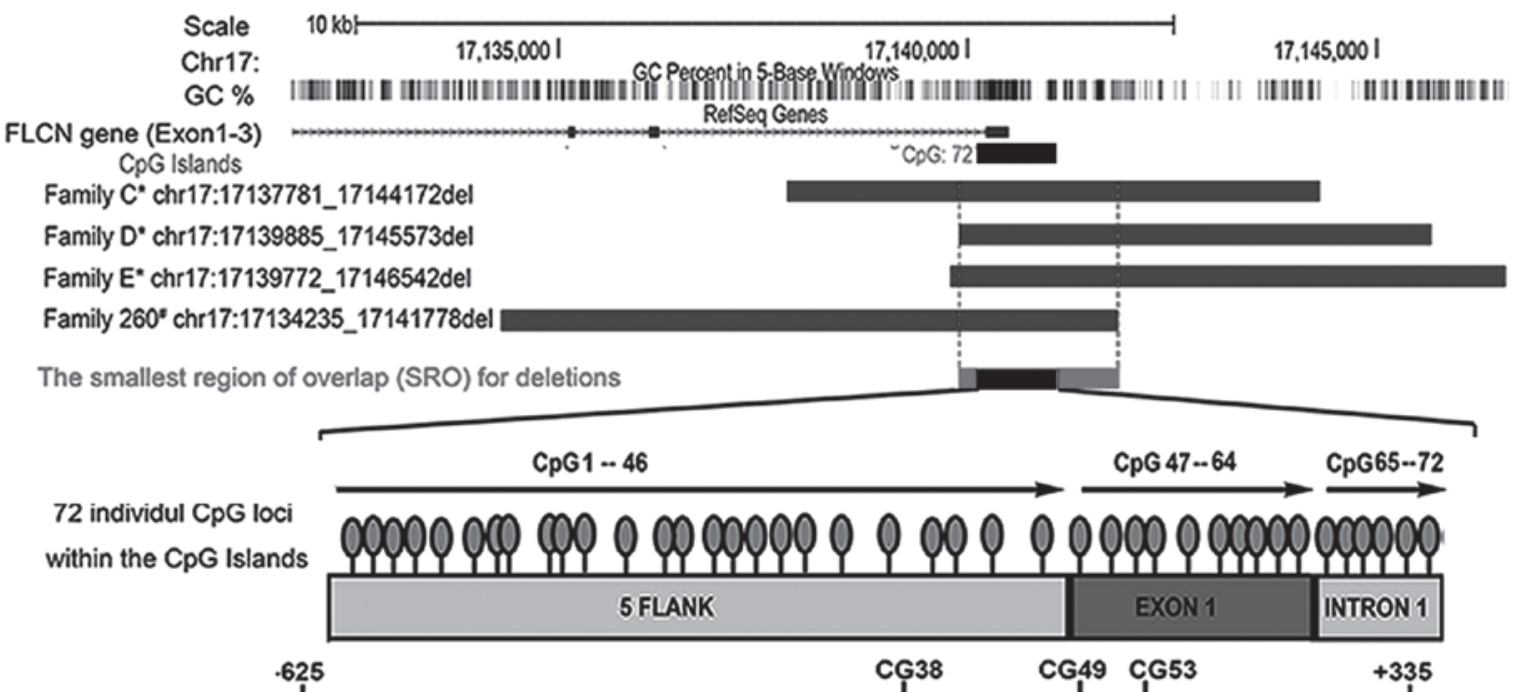

C

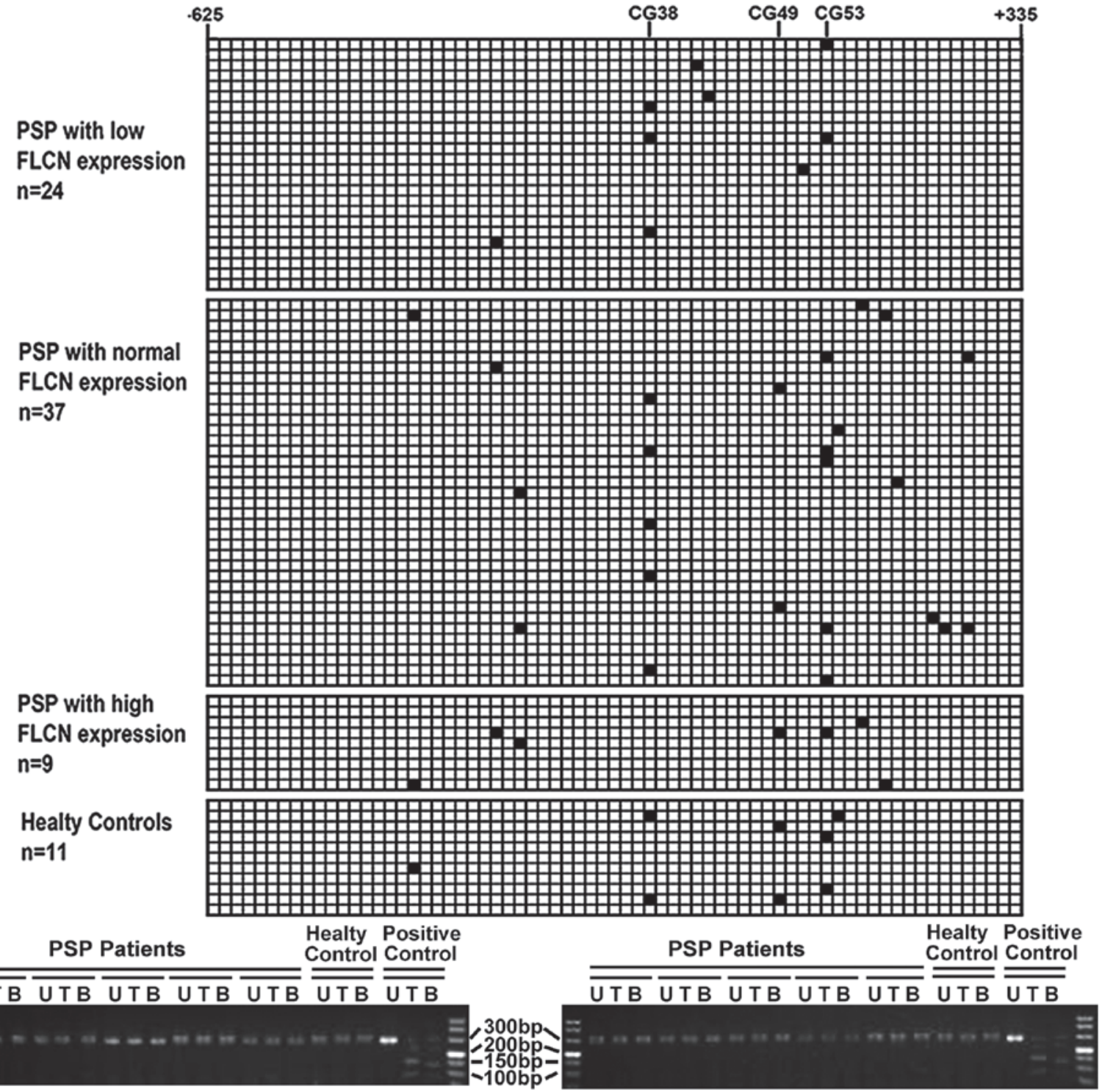

Figure 3. Methylation status of the $F L C N$ promoter in patients with PSP. (A) The genomic location of the FLCN exons 1-3, CpG Islands, deletions identified and the smallest region of overlap on chromosome 17p11.2. The information refers to the GRCh37/hg19 annotation in the UCSC and GenBank database. "Families C, D and E were previously reported (19). "Family 260 was identified in the present study. (B) The CpG Islands encompass the 5' flank, exon 1 and partial intron 1 of $F L C N$, and includes $72 \mathrm{CpG}$ loci. (C) The methylation status of 70 patients and 11 controls was assessed. Each line represents a subject analyzed by pyrosequencing, and each row represents a $\mathrm{CpG}$ locus. The black boxes indicate a methylated $\mathrm{CpG}$ island (value, $\geq 6 \%$ ) and white boxes indicate unmethylated CpG islands. No significant difference in the methylation of the $F L C N$ promoter was identified between the patients and the controls. (D) Representative data of TaqI and BstUI restriction analysis of the COBRA PCR products in the patients and the controls. The absence of digestion products indicated that the originally unmethylated restriction sites of TaqI (tcga) and Bst UI (cgcg) were lost due to bisulphite conversion. All samples tested were, therefore, unmethylated. The positive control was the universal methylated human DNA standard. PCR, polymerase chain reaction; PSP, PSP, primary spontaneous pneumothorax; U, PCR products without digestion; T, PCR products digested by Taql; B: PCR products digested by BstUI. 


\section{Acknowledgements}

The authors would like to thank the patients and family members involved in the present study. This study was supported by grants from the National Basic Research Program of China (nos. 2010CB945103 and 2009CB918704), the National Natural Science Foundation of China (nos. 81030013, 81170002 and 81471095), the Science and Technology Project of Jiangsu Province (no. BL2014053), the Ph.D. Programs Foundation of Ministry of Education of China (no. 20110091120032), the Jiangsu Province Health Department of Medicine leading talent and innovation team project (no. LJ201109) and the Foundation of Nanjing Municipal Public Health Bureau (no. ZKX11030).

\section{References}

1. Graham RB, Nolasco M, Peterlin B and Garcia CK: Nonsense mutations in folliculin presenting as isolated familial spontaneous pneumothorax in adults. Am J Respir Crit Care Med 172: 39-44, 2005.

2. Ren HZ, Zhu CC, Yang C, Chen SL, Xie J, Hou YY, Xu ZF, Wang DJ, Mu DK, Ma DH, et al: Mutation analysis of the FLCN gene in Chinese patients with sporadic and familial isolated primary spontaneous pneumothorax. Clin Genet 74: 178-183, 2008.

3. Nickerson ML, Warren MB, Toro JR, Matrosova V, Glenn G, Turner ML, Duray P, Merino M, Choyke P, Pavlovich CP, et al: Mutations in a novel gene lead to kidney tumors, lung wall defects and benign tumors of the hair follicle in patients with the Birt-Hogg-Dubé syndrome. Cancer Cell 2: 157-164, 2002.

4. Menko FH, van Steensel MA, Giraud S, Friis-Hansen L, Richard S, Ungari S, Nordenskjöld M, Hansen TV, Solly J, Maher ER, et al: Birt-Hogg-Dubé syndrome: Diagnosis and management. Lancet Oncol 10: 1199-1206, 2009.

5. Painter JN, Tapanainen H, Somer $M$, Tukiainen $P$ and Aittomäki K: A 4-bp deletion in the Birt-Hogg-Dubé gene $(F L C N)$ causes dominantly inherited spontaneous pneumothorax. Am J Hum Genet 76: 522-527, 2005.

6. Gunji Y, Akiyoshi T, Sato T, Kurihara M, Tominaga S, Takahashi K and Seyama K: Mutations of the Birt-Hogg-Dube gene in patients with multiple lung cysts and recurrent pneumothorax. J Med Genet 44: 588-593, 2007.

7. Kunogi M, Kurihara M, Ikegami TS, Kobayashi T, Shindo N, Kumasaka T, Gunji Y, Kikkawa M, Iwakami S, Hino O, et al: Clinical and genetic spectrum of Birt-Hogg-Dube syndrome patients in whom pneumothorax and/or multiple lung cysts are the presenting feature. J Med Genet 47: 281-287, 2010.

8. Abdala OA, Levy RR, Bibiloni RH, Viso HD, De Souza M and Satler VH: Advantages of video assisted thoracic surgery in the treatment of spontaneous pneumothorax. Medicina (B Aires) 61: 157-160, 2001 (In Spanish).

9. Chen YJ, Luh SP, Hsu KY, Chen CR, Tsao TC and Chen JY: Video-assisted thoracoscopic surgery (VATS) for bilateral primary spontaneous pneumothorax. J Zhejiang Univ Sci B 9: 335-340, 2008.

10. Toro JR, Pautler SE, Stewart L, Glenn GM, Weinreich M, Toure O, Wei MH, Schmidt LS, Davis L, Zbar B, et al: Lung cysts, spontaneous pneumothorax and genetic associations in 89 families with Birt-Hogg-Dubé syndrome. Am J Respir Crit Care Med 175: 1044-1053, 2007.
11. Toro JR, Wei MH, Glenn GM, Weinreich M, Toure O, Vocke C, Turner M, Choyke P, Merino MJ, Pinto PA, et al: BHD mutations, clinical and molecular genetic investigations of Birt-Hogg-Dubé syndrome: A new series of 50 families and a review of published reports. J Med Genet 45: 321-331, 2008.

12. Sahn SA and Heffner JE: Spontaneous pneumothorax. N Engl J Med 342: 868-874, 2000.

13. Grant LA, Babar J and Griffin N: Cysts, cavities and honeycombing inmultisystem disorders: Differential diagnosis and findings on thin-section CT. Clin Radiol 64: 439-448, 2009.

14. Guimaraes CV, Donnelly LF and Warner BW: CT findings for blebs and bullae in children with spontaneous pneumothorax and comparison with findings in normal age-matched controls. Pediatr Radiol 37: 879-884, 2007.

15. Jordan KG, Kwong JS, Flint J and Müller NL: Surgically treated pneumothorax. Radiologic, pathologic findings. Chest 111: 280-285, 1997.

16. Shih CH, Yu HW, Tseng YC, Chang YT, Liu CM and Hsu JW: Clinical manifestations of primary spontaneous pneumothorax in pediatric patients: An analysis of 78 patients. Pediatr Neonatol 52: 150-154, 2011.

17. Lim DH, Rehal PK, Nahorski MS, Macdonald F, Claessens T, Van Geel M, Gijezen L, Gille JJ, Giraud S, Richard S, et al: A new locus-specific database (LSDB) for mutations in the folliculin (FLCN) gene. Human Mutat 31: E1043-E1051, 2010.

18. Nahorski MS, Reiman A, Lim DH, Nookala RK, Seabra L, Lu X, Fenton J, Boora U, Nordenskjöld M, Latif F, et al: Birt Hogg-Dubé syndrome- associated FLCN mutations disrupt protein stability. Hum Mutat 32: 921-929, 2011.

19. Benhammou JN, Vocke CD, Santani A, Schmidt LS, Baba M, Seyama K, Wu X, Korolevich S, Nathanson KL, Stolle CA and Linehan WM: Identification of intragenic deletions and duplication in the FLCN gene in Birt-Hogg-Dubé syndrome. Genes chromosomes cancer 50: 466-477, 2011.

20. Khoo SK, Kahnoski K, Sugimura J, Petillo D, Chen J, Shockley K, Ludlow J, Knapp R, Giraud S, Richard S, et al: Inactivation of BHD in sporadic renal tumors. Cancer Res 63: 4583-4587, 2003.

21. Gatalica Z, Lilleberg SL, Vranic S, Eyzaguirre E, Orihuela E and Velagaleti G: Novel intronic germline $F L C N$ gene mutation in a patient with multiple ipsilateral renal neoplasms. Human pathol 40: 1813-1819, 2009.

22. Hasumi Y, Baba M, Ajima R, Hasumi H, Valera VA, Klein ME, Haines DC, Merino MJ, Hong SB, Yamaguchi TP, et al: Homozygous loss of BHD causes early embryonic lethality and kidney tumor development with activation of mTORC1 and mTORC2. Proc Natl Acad Sci USA 106: 18722-18727, 2009.

23. Hansmann T, Pliushch G, Leubner M, Kroll P, Endt D, Gehrig A, Preisler-Adams S, Wieacker P and Haaf T: Constitutive promoter methylation of BRCA1 and RAD51C in patients with familial ovarian cancer and early-onset sporadic breast cancer. Hum Mol Genet 21: 4669-4679, 2012.

24. da Silva NF, Gentle D, Hesson LB, Morton DG, Latif F and Maher ER: Analysis of the Birt-Hogg-Dubé (BHD) tumour suppressor gene in sporadic renal cell carcinoma and colorectal cancer. J Med Genet 40: 820-824, 2003.

25. El-Osta A and Wolffe AP: DNA methylation and histone deacetylation in the control of gene expression: Basic biochemistry to human development and disease. Gene Expr 9: 63-75, 2000

26. Kahnoski K, Khoo SK, Nassif NT, Chen J, Lobo GP, Segelov E and Teh BT: Alterations of the Birt-Hogg-Dubé gene (BHD) in sporadic colorectal tumours. J Med Genet 40: 511-515, 2003. 